\title{
Osteopathic Approach to the Treatment of a Patient With Idiopathic Iliohypogastric Neuralgia
}

David B. Fuller, DO

From the Department of Osteopathic Manipulative Medicine at Philadelphia College of Osteopathic Medicine in Pennsylvania.

Financial Disclosures: None reported.

Support: None reported.

Address correspondence to David B. Fuller, DO,

2075 Paper Mill Road, Huntingdon Valley, PA 19006-5815.

Email: davidfu@pcom.edu Submitted May 27, 2020; revision received July 1, 2020; accepted July 14, 2020.
Iliohypogastric neuralgia is an uncommon etiology of lower abdominal pain caused by entrapment of the iliohypogastric nerve. Conventional management consists of medications, injections, and surgery; previous literature has not explored the use of osteopathic manipulative medicine for management of iliohypogastric neuralgia. Here, the author discusses the case of a 72-year-old woman who presented with 2 years of right lower abdominal pain, having failed multiple treatments, including exploratory laparoscopy and appendectomy. Following management of the patient's somatic dysfunctions with osteopathic manipulative treatment and a heel lift, her iliohypogastric neuralgia was significantly improved.

$J$ Am Osteopath Assoc. 2020;120(12):907-912. Published online October 21, 2020. doi:10.7556/jaoa.2020.150

Keywords: abdominal pain, iliohypogastric neuralgia, OMM, OMT, osteopathic manipulative treatment, short leg syndrome

I diopathic iliohypogastric neuralgia is an uncommon and often underrecognized cause of lower abdominal pain. ${ }^{1,2}$ Neuropathy of the iliohypogastric nerve is a reported complication following lower abdominal and pelvic surgeries, although less common than ilioinguinal neuropathy. ${ }^{3}$ The iliohypogastric nerve arises from the $12^{\text {th }}$ thoracic, first lumbar, and sometimes the second lumbar nerves as part of the lumbar plexus and runs laterally, posterior to the psoas, crossing the anterior surface of the quadratus lumborum, wrapping forward to reach the iliac crests, where it perforates the transversalis fascia and the transversus muscle, medial to the anterior superior iliac spine (ASIS). It then divides into an anterior (hypogastric branch) and a lateral (iliac) branch. ${ }^{47}$ The anterior branch is a sensory nerve that pierces the internal oblique muscle medial to the ASIS (as well as the fascia along the posterior and anterior aspects of the muscle), running between the internal and external oblique muscles until it finally pierces the aponeurosis of the external oblique above, and lateral to, the superficial inguinal ring. ${ }^{5-7}$ This nerve supplies the anterior abdominal wall of the unilateral hypogastric region. The lateral (iliac) branch is also a sensory nerve, immediately piercing the internal and external oblique muscles near the ASIS, supplying the skin over the outer buttock and lateral hip (Figure 1).

The iliohypogastric nerve can be compressed within the narrow anatomic canals and openings through which it runs, including where the nerve pierces through fascia and muscle, beneath tight ligaments, fibrous bands, or sutures. ${ }^{8,9}$ While the most common causes of painful iliohypogastric neuropathies are due to abdominal and anterior pelvic wall processes, the nerve can be injured posteriorly in the lumbar plexus. ${ }^{5,6}$

Diagnosis of iliohypogastric neuralgia is usually made clinically, based on the pattern of pain and/or sensory changes following the distribution of the nerve. Diagnosis can be 


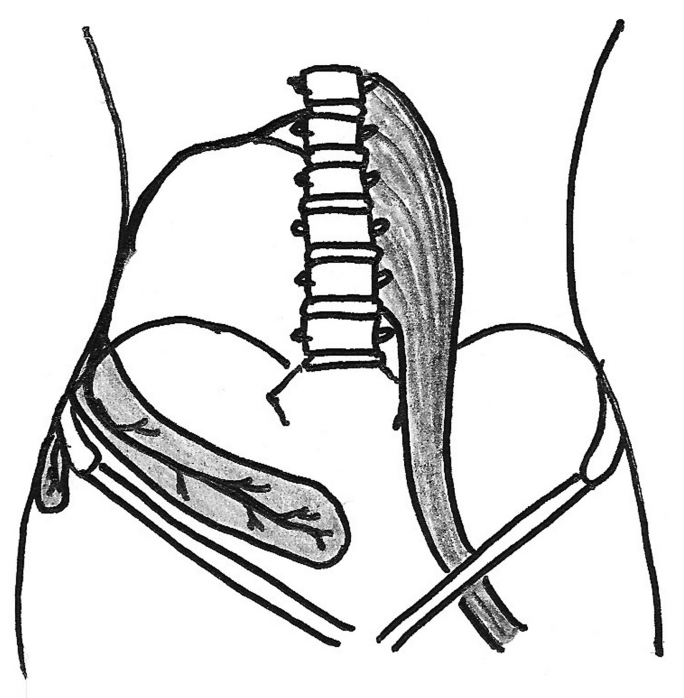

Figure 1.

lliohypogastric nerve anatomy.

confirmed with a nerve block. ${ }^{6,10}$ Electromyography studies usually have insufficient sensitivity for this diagnosis, although they may be useful to rule out radiculopathy or plexopathy. ${ }^{5,9,11}$ Conventional management consists of medications, nerve blocks, neurolytic procedures, surgical exploration, and neurectomy. ${ }^{6,11}$ While osteopathic manipulative treatment (OMT) has been used to manage entrapment neuropathies ${ }^{11,12}$ including meralgia paresthetica, which involves entrapment of the lateral femoral cutaneous nerve - there does not appear to be any published literature involving the use of OMT in the treatment of iliohypogastric neuralgia. Here, we report successful management of iliohypogastric neuralgia with OMT and a heel lift to address postural decompensation effects due to short leg syndrome.

\section{Report of Case}

A 72-year-old woman who was a retired figure skating coach presented to the office in August 2017 upon referral by her family physician for OMT evaluation and treatment of her severe, burning, right-lower-quadrant abdominal pain of unknown etiology for the prior 2 years. There were no clear triggers for the patient's pain; it would occur randomly and would often wake her at night. The pain was frequent, occurring both during the day and at night, with a duration of 20-60 minutes per episode. The pain radiated from the anterior, superior aspect of the patient's right hip across her right lower abdomen; it did not cross the midline. Rarely, the pain would radiate posteriorly from the ASIS, wrapping around the lateral right hip. Usually, the pain was in the right lower quadrant of the abdomen, immediately above the inguinal canal. It was not tender to the touch, nor reproduced by palpation.

The patient had previously undergone extensive evaluations by her family physician, a gastroenterologist, a gynecologist, and a general surgeon. None of these previous examinations revealed the cause of her pain. Pelvic ultrasound was normal. Computed tomography (CT) of the abdomen and pelvis showed hepatic steatosis and possible diverticulosis. Her colonoscopy was reported as normal. X-ray of the patient's lumbar spine showed a mild lumbar lateral curve, convex left, with generalized multilevel disc space narrowing. Four months prior to her presentation, she had undergone an exploratory laparoscopy and appendectomy. She felt some improvement for 2 months postoperatively, then the pain returned, becoming worse than before surgery.

Her past medical history included a mild stroke 10 years earlier (with no residual deficits), hypertension, hyperlipidemia, narrow angle glaucoma, gastroesophageal reflux disease, benign breast mass, rotator cuff tear, cataracts, macular degeneration, and osteopenia. The patient's surgical history included rotator cuff repair, cataract extraction and lens implantation, and breast biopsy. Her current medications included ramipril, esomeprazole, hydrochlorothiazide, naproxen, atorvastatin, hyoscyamine, and low-dose aspirin. A review of the patient's symptoms showed no fatigue, fever, night sweats, ear pain, eye pain, sore throat, cough, wheezing, chest pain, palpitations, diarrhea, constipation, urinary incontinence, cold intolerance, 
extremity numbness, extremity weakness, rash, easy bleeding, or seasonal allergies. She did have occasional low back pain but reported none in the recent past.

Physical examination showed a woman who appeared in good health, with appropriate weight and with normal findings, except musculoskeletal structural findings described here. Her breathing was easy and unlabored. She had no cyanosis and had normal pulses at the dorsalis pedis arteries bilaterally. Her neurologic examination was symmetrical, with normal gait and station; deep tendon reflexes of the lower extremity were normal, as was the motor examination of her lower extremities. Her lower extremity examination showed normal appearance, with no muscle wasting, atrophic changes, or abnormal dermatological findings. Her hip examination showed full, normal, passive range of motion bilaterally, with no tenderness on examination. Her Patrick test and hip impingement tests were negative. She had no tenderness at the anterior or lateral hips. Her abdomen was normal to palpation with no tenderness on examination. Her osteopathic structural findings included decreased mobility of the entire lumbar spine with a lateral curve that was convex left, L5 extended, rotated left, sidebent left; a bilaterally extended sacrum; marked anterior right innominate rotation; bilaterally restricted psoas muscles; and fascial restriction of the right lower abdomen and linea alba. A presumptive diagnosis was made of right iliohypogastric neuralgia with somatic dysfunction of the lumbar, pelvis, sacral, and lower extremity areas.

The patient was treated with OMT at that time, which included balanced ligamentous tension, ligamentous articular strain, myofascial release, muscle energy, and Fulford percussor techniques. Specifically, the right anterior innominate dysfunction was treated with the Fulford percussor technique, then ligamentous articular strain OMT, followed by muscle energy technique. The patient's lumbar spine restriction (L1-L5) was treated with the Fulford percussor technique followed by specific treatment of the L5 vertebral dysfunction with the Fulford percussor technique and balanced ligamentous tension. Her psoas dysfunction was treated with the muscle energy technique. Her sacroiliac dysfunction was treated with ligamentous articular strain OMT. The fascial restrictions of the right lower abdomen and linea alba were treated with indirect myofascial release and ligamentous articular strain techniques. She was then taught home exercises involving pelvic rotations in 4 directions ("pelvic clock") and instructed to perform them daily. She tolerated the treatment and exercises well.

The patient returned 1 week later and reported significant improvement in her abdominal pain. She was performing home exercises daily without difficulty. Her pattern of somatic dysfunctions was similar, but with less asymmetry, and she was treated again with similar OMT techniques. At this visit, she was prescribed over-the-counter alpha lipoic acid, 200mg daily, to facilitate improved function of the iliohypogastric nerve. While this supplement has not been studied with iliohypogastric neuralgia, it has been shown to be helpful with diabetic peripheral neuropathy. ${ }^{14}$

Over the next 3 months, the patient was evaluated and treated with an osteopathic manipulative medicine (OMM) diagnostic and therapeutic approach at 2-week intervals, with a steady improvement of symptoms. At first, her symptoms would improve for the week following OMT, then gradually recur. Her pain-free interval increased as the number of total OMT visits increased. By the end of 3 months, and a total of 10 sessions, she had very little abdominal pain, reporting mostly only paresthesias in the right lower abdomen of short duration. She subjectively noted that her symptoms were $80 \%$ improved. At that time, she was performing 2 additional daily, supine pelvic and hip home exercises, which involved maintaining a posterior pelvic tilt with hip adductor lengthening and psoas lengthening, as well as continuing to take the alpha lipoic acid, also daily.

During the fourth month, the patient had a flare of right lower quadrant abdominal pain that coincided with increased standing for 2 weeks while preparing her home for the holidays. She was still being evaluated 


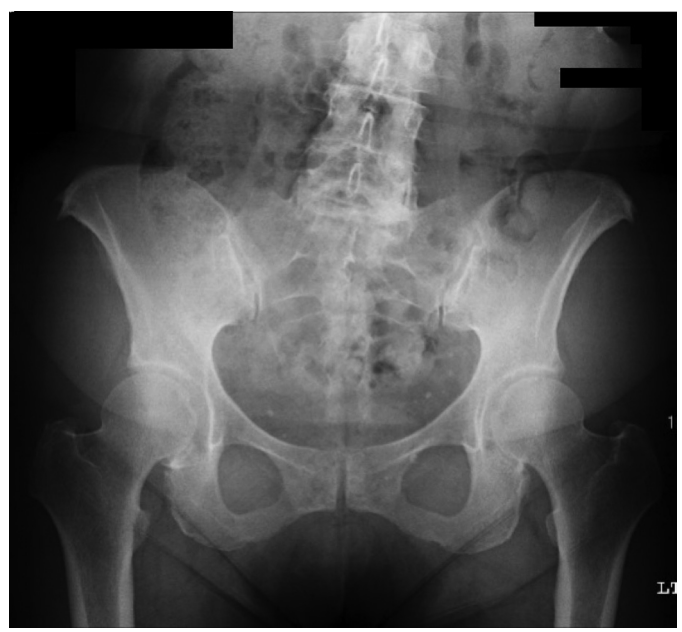

Figure 2.

This standing, anteroposterior xray of the pelvis demonstrates the patient's sacral base unleveling consistent with short leg syndrome.

and treated with OMM twice monthly. A standing anteroposterior xray of the pelvis was ordered to investigate possible short leg postural mechanics. It showed an inferior left femoral head with sacral base unlevelling. She was prescribed a $3 \mathrm{~mm}$ heel lift for her left shoe. Her right-sided pelvic side shift improved when standing with the lift in place. However, she did not start using the lift regularly (Figure 2).

One week later, the patient developed pain radiating down to her posterior right thigh and lateral knee, suspicious for a radicular process. Magnetic resonance imaging (MRI) of the lumbar spine was performed, which showed multilevel lumbar degenerative changes, including L1-L2 bilateral foraminal and lateral disc osteophyte complexes with minimal foraminal narrowing. She followed up at the office 1.5 weeks later, after using the heel lift regularly for 2 weeks. The patient reported being pleased with the significant improvement in both her abdominal pain and lower extremity pain.

The patient was subsequently seen at monthly intervals, treated with OMT each visit, and her right lower quadrant abdominal pain was almost completely resolved; reported a few seconds of pain on rare occa- sions only. She still had some pelvic sideshift toward the long leg side and a mildly positive standing flexion test; therefore, her heel lift was increased to $5 \mathrm{~mm}$, which improved her short leg mechanics to the point of normalizing her pelvic side shift and standing flexion test findings.

The patient has continued to do well since that time (February 2018), with 1 significant recurrence of right lower quadrant pain that coincided with altered gait, due to wearing an ankle immobilizer for an ankle fracture that required the use of a cane when walking.

She was seen once per month for 5 months, had a single treatment 3 months later, and then was seen twice more at 6-month intervals, for a total of 10 treatments in addition to the previous 10 . In her last year of follow-up, the patient reported having right lower quadrant pain only when she failed to wear her heel lift for 1 to 2 days. She also noted occasional, intermittent discomfort in the right lower abdominal wall when lying on her right side, which would resolve with change in position.

\section{Discussion}

This case illustrates 2 significant OMM treatments for this patient's iliohypogastric neuralgia - OMT and heel lift therapy.

The patient's symptoms of right lower quadrant pain with occasional right hip/buttock pain, which she had for 2 years prior to presentation, responded quickly to OMT, with a self-reported $80 \%$ reduction in symptoms after 6 sessions over 2 months. The fact that the pain was primarily medial to the ASIS and radiated across her right lower abdomen in the distribution of the anterior (hypogastric) branch of the iliohypogastric nerve, as well as occasional lateral radiation (iliac branch), suggested compression of the nerve near the ASIS, where it perforated multiple layers of fascia and muscle. This was likely caused by a myofascial strain related to underlying somatic dysfunctions, especially the markedly anterior rotation dysfunction of the right innominate. 
The patient's initial symptoms improved to a plateau of $80 \%$ improvement with OMT, home exercises, and possible mild benefit from alpha lipoic acid supplementation. The patient's further improvement coincided directly with initiation of heel lift therapy to address her short leg syndrome mechanics, demonstrating a clear response to this intervention. It also correlated with normalization of her standing postural findings, which included pelvic side shift. ${ }^{15}$ The fact that she had intermittent radicular symptoms and neuroforaminal stenosis at L1-L2 on MRI, complicating her lumbar convex left curve, as well as multilevel degenerative changes, suggests that there may have been a "double crush" component involving the origin of the nerve fibers as they travelled in the nerve roots of T12-L2. This likely neuroforaminal stenosis component was reinforced by the fact that as she was improving, the patient experienced right lower abdominal pain and paresthesias when she would lie on her right side, likely caused by right sidebending of the thoracolumbar junction that further narrowed the stenosed neuroforamen at that level.

There is literature ${ }^{15-17}$ supporting the use of heel lift therapy for patients with leg length discrepancies that cause sacral base unlevelling ("short leg syndrome"). An osteopathic approach to heel lift therapy for patients with short leg syndrome always involves OMT. ${ }^{16}$ While there is mention of piriformis involvement with short leg syndrome that can cause sciatica, ${ }^{17}$ there does not appear to be any previous literature demonstrating the use of heel lift therapy in patients with peripheral neuropathies involving double crush syndromes.

\section{Conclusion}

This patient with chronic iliohypogastric neuralgia responded well to an OMM approach with OMT and heel lift therapy, which improved her structure and function and brought relief for this 2-year problem. Patients with iliohypogastric neuralgia may have components of myofascial restriction, especially related to abdominal wall myofascial structures affected by underlying somatic dysfunction, causing or aggravating entrapment of the iliohypogastric nerve. They may also have a component of "double crush" syndrome involving the lumbar spine due to postural imbalance, likely aggravating underlying neuroforaminal stenosis, as well as fascial strain patterns. It is worthwhile to look for and treat underlying components of somatic dysfunction and postural imbalance in patients with iliohypogastric neuralgia; an OMM approach, including OMT and heel lift therapy, is a good option to consider in appropriate patients.

\section{Author Contributions}

The author provided substantial contributions to conception and design, acquisition of data, or analysis and interpretation of data; the author drafted the article or revised it critically for important intellectual content; the author gave final approval of the version of the article to be published; and the author agrees to be accountable for all aspects of the work in ensuring that questions related to the accuracy or integrity of any part of the work are appropriately investigated and resolved.

\section{References}

1. Amin N, Krashin D, Trescot A. llioinguinal and iliohypogastric nerve entrapment: abdominal. In: Trescott A, ed. Peripheral Nerve Entrapments: Clinical Diagnosis and Management. 1st ed. Switzerland: Springer; 2016:413-424.

2. Kim D, Murovic J, Tiel R, Kline D. Surgical management of 33 ilioinguinal and iliohypogastric neuralgias at Louisiana State University Health Sciences Center. Neurosurgery. 2005;56(5):1013-1020.

3. Shahu K, Ramlagun D, Chen S, Liu L. Neuralgia due to iliohypogastric nerve injury after inguinal hernioplasty: a case report. BMC Surgery. 2018;18(59). doi:10.1186/s12893-018-0391-67

4. Brazis P, Masdeu J, Biller J. Chapter 2: Peripheral nerves. Section: lliohypogastric, ilioinguinal, and genitofemoral nerves. In: Localization in Clinical Neurology, $7^{\text {th }}$ Ed. LWW; 2016. E-book.

5. Rassner L. Lumbar plexus nerve entrapment syndromes as a cause of groin pain in athletes. Curr Sports Med Rep. 2011;10(2):115-120. doi:10.1249/JSR.0b013e318214a0457

6. Pick T, Howden R, ed. Gray's Anatomy. 15th ed. New York: Bounty Books. 1977:782.

7. Moore K. Clinically Oriented Anatomy. 2nd ed. Baltimore: Williams \& Wilkins;279.

8. Hoffman B, Schorge J, Halvorson L, Hamid C, Corton M, Shaffer J. Williams Gynecology. 4th ed. E-book. Chapter 12: Pelvic pain. Section: Neurologic Etiologies. Subsection: Anterior abdominal wall nerve entrapment syndrome. McGraw-Hill Education. 2020.

9. Hawksworth D, Dellon L, Herati A. llioinguinal and iliohypogastric neuralgia as an etiology of bladder pain syndrome. Urol Case Rep. 2020:28. doi:10.1016/j.eucr.2019.1010567

10. Flaherty A, Rost N. The Massachusetts General Hospital Handbook of Neurology. 2nd ed. E-book. Lipppincott, Williams, \& Wilkins. 2007. 
11. Elkiss M, Heinking K, Hruby R, Fraix M, Giusti R. Osteopathic considerations in neurology. In: Seffinger M, ed. Foundations for Osteopathic Medicine. 4th ed. Philadelphia: Wolters Kluwer; 1275-1280.

12. Torrents M, Malik R. Meralgia paresthetica: a model approach Osteo Fam Phys. 2018;10(5):10-15.

13. Schiowitz S. Chapter 102: Practical applications and case histories of the lower extremities. In: DiGiovanna EL, Amen JA, Burns DK, eds. An Osteopathic Approach to Diagnosis and Treatment, $4 \mathrm{e}$ ed. Wolters Kluwer; 2021.

14. Han T, Bai J, Liu W, Hu Y. A systematic review and meta-analysis of alpha lipoic acid in the treatment of diabetic peripheral neuropathy. Eur J Endocrinology. 2012;167(4):465-71. doi:10.1530/EJE-12-05557
15. Nelson K, Mnabhi A. The patient with back pain: short leg syndrome and postural balance. In: Nelson K, ed. Somatic Dysfunction in Osteopathic Family Medicine, 2nd ed. Philadelphia: Wolters Kluwer; 415-432.

16. Kuchera M. Postural considerations in osteopathic diagnosis and treatment. In: Seffinger M, ed. Foundations for Osteopathic Medicine. 4th ed. Philadelphia: Wolters Kluwer; 697.

17. Schiowitz S, DiGiovanna E, DiGiovanna J. Chapter 58: Gait and postural considerations. In: DiGiovanna EL, Amen JA, Burns DK, eds. An Osteopathic Approach to Diagnosis and Treatment, $4 \mathrm{e}$ ed. Wolters Kluwer; 2021.

() 2020 American Osteopathic Association 